\title{
Improved assessment of aortic hemodynamics by $k-t$ accelerated dual-venc 4D flow MRI in pediatric patients
}

\author{
Susanne Schnell ${ }^{1 *}$, Michael J Rose ${ }^{2,1}$, Can Wu ${ }^{1,3}$, Julio Garcia', Joshua D Robinson ${ }^{4}$, Michael Markl ${ }^{1,3}$, \\ Cynthia K Rigsby ${ }^{2}$
}

From 19th Annual SCMR Scientific Sessions

Los Angeles, CA, USA. 27-30 January 2016

\section{Background}

Bicuspid aortic valve (BAV) is often associated with aortic stenosis, resulting in high velocity blood flow in the ascending aorta, with slow flow areas in the dilated aorta or adjacent vessels. Similarly, Marfan syndrome patients develop aortic root dilatation and regurgitation with both high and low velocity blood flow. 4D flow MRI is typically acquired with a velocity encoding (venc) gradient adjusted to the estimated peak velocity. As the velocity noise is directly related to the velocity sensitivity $(\sigma v \sim v e n c)$, a high venc can substantially limit the assessment of low flow velocities $(v<<v e n c)$. We therefore developed a fully integrated low- and high-venc PC-MRI in a single measurement (dual-venc) to avoid aliasing and improve velocity-to-noise ratio (VNR).

\section{Methods}

In addition to standard clinical contrast enhanced cardiac MRI, $k-t$ GRAPPA accelerated dual-venc 4D flow MRI was acquired with full volumetric coverage of the ascending (AAo) and descending (DAo) aorta in 4 Marfan syndrome patients (age $=16.3 \pm 1.9 \mathrm{yrs}, 1$ female, low-venc $=81 \mathrm{~cm} /$ $\mathrm{s}$, high-venc $=200 \mathrm{~cm} / \mathrm{s})$ and $1 \mathrm{BAV}$ patient $(\mathrm{age}=20 \mathrm{yrs}$, male, low-venc $=125 \mathrm{~cm} / \mathrm{s}$, high-venc $=350 \mathrm{~cm} / \mathrm{s}$ ) on a 1.5T Siemens MAGNETOM Aera MRI scanner with the following imaging parameters: $\mathrm{R}=5, \mathrm{TE} / \mathrm{TR}=2.93 \pm$ $0.08 / 5.46 \pm 0.08 \mathrm{~ms}$, voxel size $=1.9 \times 1.9 \times 2.0 \mathrm{~mm}^{3}$. Background correction was performed separately of the low- and high-venc phase images. High-venc data were used for complete anti-aliasing of the low-venc data while maintaining the favorable VNR of the low-venc data.

'Dept. of Radiology, Northwestern University, Feinberg Medical School,

Chicago, IL, USA

Full list of author information is available at the end of the article
A pseudo-complex MRA was derived from the 4D flow data and used to segment the angiogram using commercial software (MIMICS, Materialize). Velocity noise was estimated in static tissue using region of interest analysis. Velocity maximum intensity projection maps (MIPs) were calculated and used to determine peak velocities in the overall aorta and in the three sub-segments AAo, Arch and DAo. In addition, blood flow was visualized using streamlines within the segmented vessels during peak systole.

\section{Results}

Velocity noise was significantly improved in the dualvenc $4 \mathrm{D}$ flow data by $230 \% \pm 20 \%$ compared to the high-venc velocity scan for the four Marfan patients, which corresponded with the high-venc/low-venc ratio of 2.5. In the BAV patient, velocity noise was improved by $276 \%$ in the dual-venc compared to the high-venc scan (high-venc/low-venc ratio=2.4). Peak velocity showed significant correlation between high- and dual-venc results in the AAo $\left(R^{2}=0.85, \mathrm{p}=0.04\right)$ and Arch $\left(\mathrm{R}^{2}=0.95, \mathrm{p}=\right.$ $0.006)$ using velocity MIP analysis. Streamline comparison showed substantially improved qualitative visualization of aortic blood flow patterns and more coherent streamlines especially in slow flow areas and no aliasing artifacts in areas that were aliased in the low-venc images.

\section{Conclusions}

This feasibility study demonstrates the potential of $k-t$ accelerated dual-venc 4D flow MRI to improve the assessment of aortic hemodynamics with large dynamic range while maintaining the scan time of standard 4D flow MRI. 


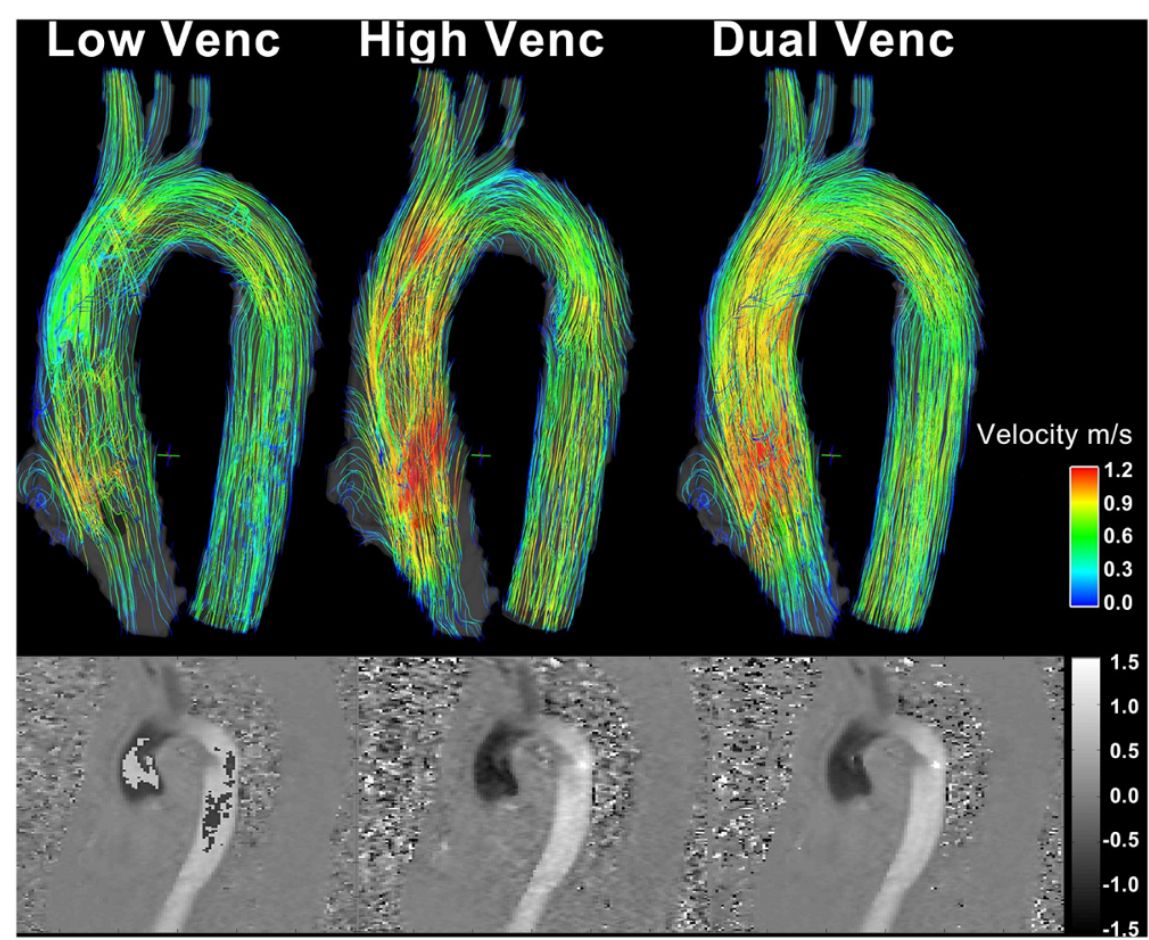

Figure 1

\section{Authors' details}

'Dept. of Radiology, Northwestern University, Feinberg Medical School,

Chicago, IL, USA. 'Pediatric Radiology, Lurie Children's Hospital of Chicago, Chicago, IL, USA. ${ }^{3}$ McCormick School of Engineering, Northwestern

University, Evanston, IL, USA. ${ }^{4}$ Pediatrics-Cardiology, Lurie Children's Hospital

of Chicago, Chicago, IL, USA.

Published: 27 January 2016

doi:10.1186/1532-429X-18-S1-096

Cite this article as: Schnell et al.: Improved assessment of aortic

hemodynamics by $k-t$ accelerated dual-venc $4 \mathrm{D}$ flow MRI in pediatric patients. Journal of Cardiovascular Magnetic Resonance 2016 18(Suppl 1):

096.

Submit your next manuscript to BioMed Central and take full advantage of:

- Convenient online submission

- Thorough peer review

- No space constraints or color figure charges

- Immediate publication on acceptance

- Inclusion in PubMed, CAS, Scopus and Google Scholar

- Research which is freely available for redistribution 